\title{
Three-dimensional force measurements in optical tweezers formed with high-NA micromirrors
}

\author{
Fabrice Merenda, ${ }^{1, *}$ Mathieu Grossenbacher, ${ }^{1}$ Sylvia Jeney, ${ }^{2}$ László Forró, ${ }^{2}$ and René-Paul Salathé ${ }^{1}$ \\ ${ }^{1}$ Advanced Photonics Laboratory, École Polytechnique Fédérale de Lausanne, \\ Station 17, CH-1015 Lausanne, Switzerland \\ ${ }^{2}$ Laboratory of Nanostructures and Novel Electronic Materials, École Polytechnique Fédérale de Lausanne, \\ Station 3, CH-1015 Lausanne, Switzerland \\ *Corresponding author: fabrice.merenda@a3.epfl.ch
}

Received January 16, 2009; accepted February 10, 2009; posted February 25, 2009 (Doc. ID 106429); published March 25, 2009

The three-dimensional trap stiffness of optical tweezers formed with high-NA micromirrors is investigated by back-focal-plane interferometry and power spectrum analysis. Normalized stiffness values of $\kappa_{x y} / P_{\text {trap }}$ $=1.2(\mu \mathrm{N} / \mathrm{m}) / \mathrm{mW}$ and $\kappa_{z} / P_{\text {trap }}=0.52(\mu \mathrm{N} / \mathrm{m}) / \mathrm{mW}$ in the transverse and axial directions, respectively, have been measured for polystyrene spheres with a radius of $1.03 \mu \mathrm{m}$. Compared with high-NA microscope objectives, micromirrors achieve much better trapping performances, particularly in the axial direction. (C) 2009 Optical Society of America

OCIS codes: $350.4855,130.3990,350.3950,230.4040,220.1230$.

Optical tweezers (OT) [1] have found broad applications in biology and physics, e.g., for the mechanical characterization of single biomolecules [2,3], for fundamental studies on Brownian motion and thermodynamics at the microscopic scale [4,5], or for in situ viscometry [6]. The tight focusing of the laser beam, necessary for creating stable three-dimensional (3D) OT, is usually achieved with a high-NA microscope objective. However, the cost, bulkiness, and short working distance of these objectives severely limits the conceivable application range of OT. We have recently proposed the use of miniaturized high-NA parabolic mirrors, integrated at the level of a microfluidic chip, for simultaneous multiple optical trapping and fluorescence detection [7]. Such micromirror OT (MOT) are among the few existing integrated geometries capable of 3D optical trapping that include fiber-based optical traps [8-10], diode lasers monolithically integrated in microfluidics [11], and microfabricated water-immersion zone plates [12]. MOT were already shown to withstand large escape forces in microfluidic flows with beads as large as $9.33 \mu \mathrm{m}$ in diameter. However, the most important aspect for applications where OT are used as force transducers is the trap stiffness achievable with micrometer-sized spheres. In this Letter, we present precise measurements of the 3D trap stiffness generated by MOT, using back-focal-plane interferometry and power spectrum analysis.

Micromirrors were fabricated using a molding technique described in detail in [7]. Briefly, a fusedsilica microlens array (Süss MicroOptics, Switzerland) coated with a 50-nm-thick gold layer is immersed in UV epoxy (NOA65, $n=1.51$ at $1064 \mathrm{~nm}$ ) onto a 1-mm-thick glass slide. After polymerization, the microlens array is ripped off the glass slide, leaving its inverse structure coated with gold on the latter. The concave mirrors are further filled with the same UV epoxy and protected with an $80-\mu \mathrm{m}$-thick coverglass glued on top. Each micromirror has a diameter of $240 \mu \mathrm{m}$ and a radius of curvature of
$350 \mu \mathrm{m}$, yielding an effective NA of 0.93 . The foci of micromirrors are located approximately $10 \mu \mathrm{m}$ above the protective coverglass. A glass flow chamber assembled around the slide with micromirrors allows conveying particles to the trapping area using a fluidic system.

Laser light from an ytterbium fiber laser emitting in a linearly polarized $\mathrm{TEM}_{00}$ mode (IPG Photonics, YP-1064LP, $\lambda=1064 \mathrm{~nm}$ ) is resized by an afocal telescope to a Gaussian diameter $\left(1 / e^{2}\right.$ level $)$ approximately five times larger than the diameter of a single micromirror. As illustrated in Fig. 1, this laser beam hits a $5 \mathrm{~mm}$ large nonpolarizing $50 \%$ beam splitter cube placed on top of the fluidic chamber, allowing half of the laser power to strike upon the micromirrors at normal incidence. A long working distance microscope objective (Leitz-Wetzlar $\mathrm{H} 32 \times, \mathrm{NA}=0.6$, $\infty / 1.80$ Quarzglas, Germany) positioned on top of a

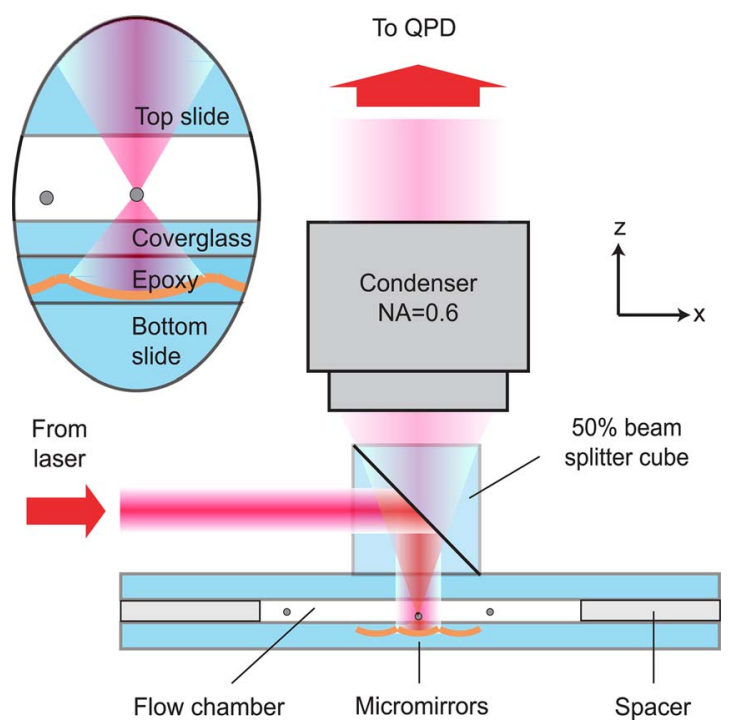

Fig. 1. (Color online) Schematic representation of the optical setup employed for forward light scattering detection from an MOT. The inset shows the vertical structure of the fluidic device embedding micromirrors. 
selected micromirror in the array collects scattered and unscattered light across the same beam splitter cube, while the objective's back focal plane is imaged onto an InGaAs quadrant photodiode (G6849, Hamamatsu, Japan). The photodiode signals encoding the $3 \mathrm{D}$ position of the particle $[13,14]$ are amplified analogically (Öffner Electronics, Germany) and digitized to 16 bits (LTT-184, LTT GmbH, Germany) at a frequency of $104 \mathrm{kHz}$ during $20.2 \mathrm{~s}$. The measured trajectories in the $x, y$, and $z$ directions are Fourier transformed to obtain the power spectral densities $P(f)$, that are subsequently gathered into blocks to ensure normal distribution $\left(n_{b}=200\right.$ points per block) [15]. A precise control of the laser power in the trap is crucial for the comparison with values of trap stiffness previously reported in the literature. It is calculated from the measured optical power and Gaussian beam width at the beam splitter cube, and accounts for the power transmission of $93 \%$ characteristic for micromirrors [7]. The employed polystyrene (PS) microspheres (Polysciences, Inc., USA, radius of $a=1.03 \mu \mathrm{m}$ ) were suspended in pure distilled water at a concentration of $\sim 10^{6}$ microspheres $/ \mathrm{ml}$.

The theoretical expectation for $P(f)$, including hydrodynamic memory effects that are nonnegligible in our experimental conditions [16], is given by BergSørensen and Flyvbjerg [15],

$$
P(f)=\frac{D}{2 \pi^{2}} \frac{\left[1+\left(f / f_{v}\right)^{1 / 2}\right]}{\left(f_{c}-\frac{f^{3 / 2}}{f_{v}^{1 / 2}}-\frac{f^{2}}{f_{m}}\right)^{2}+\left(f+\frac{f^{3 / 2}}{f_{v}^{1 / 2}}\right)^{2}} .
$$

This equation is fitted to the blocked experimental $P(f)$ with the particles's diffusion constant $D$ and the corner frequency $f_{c}$ as free parameters. The latter gives direct access to the trap's spring constant $\kappa$ $=2 \pi \gamma f_{c}$, where $\gamma=6 \pi \eta a$ is the friction coefficient depending on the kinematic viscosity $\eta$ of the fluid and the radius $a$ of the trapped sphere. The characteristic frequencies $f_{\nu}=\eta /\left(2 \pi a^{2} \rho\right)$ (where $\rho$ is the fluid density) and $f_{m}=3 \mathrm{\eta a} / \mathrm{m}$ (where $m$ is the mass of the trapped sphere) are described in [15]. The detector sensitivity, relating the photodiode voltage signals to the absolute bead displacements, is calibrated by comparing the theoretical diffusion constant $D$ $=k_{B} T / \gamma$ with the diffusion constant fitted to noncalibrated signals. Finally, aliasing artifacts are avoided by limiting the fitting procedure to one tenth of the sampling frequency [15].

Figure 2 illustrates experimental power spectral densities measured on an MOT. For clarity, only the power spectra in the $x$ direction (cross) and the $z$ direction (plus) are shown, as the $x$ and $y$ power spectra are almost overlapping. The background noise levels acquired while the trap was empty are also shown (lower curves), demonstrating that the signal-tonoise ratio is well above 10 all over the shown frequency range. Continuous curves are fits to Eq. (1), whose quality is supported by the calculated fractional standard deviation of $7 \%$ between $20 \mathrm{~Hz}$ and $10.4 \mathrm{kHz}$, in agreement with the expected fractional deviation of $1 / \sqrt{n_{b}}$ [15]. In the data shown on Fig. 2

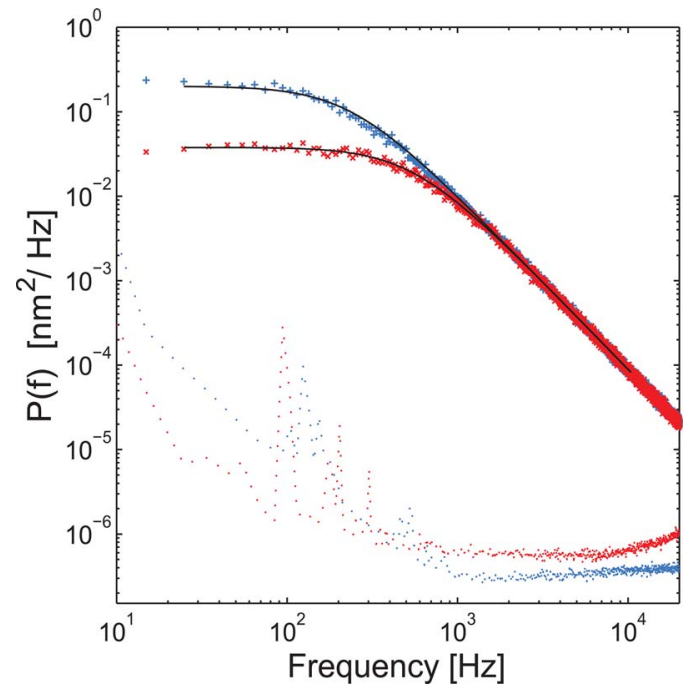

Fig. 2. (Color online) Blocked $P(f)$, in the $x$ (cross) and $z$ (plus) directions, measured on an MOT when trapping PS spheres with radius $a=1.03 \mu \mathrm{m}$. Continuous black curves are fits to Eq. (1). Lower traces show the instrument background noise levels.

the cut-off frequencies are $531 \mathrm{~Hz}$ in the $x$ direction and $230 \mathrm{~Hz}$ in the $z$ direction, corresponding to restoring spring constants of $\kappa_{x}=65 \mu \mathrm{N} / \mathrm{m}$ and $\kappa_{z}$ $=28 \mu \mathrm{N} / \mathrm{m}$ achieved with an optical power of $P_{\text {trap }}$ $=55 \mathrm{~mW}$. Position fluctuations in the MOT, calibrated through the fitting procedure, are shown on the 2D histograms of Fig. 3 . The trapping potential is almost symmetric in the $x-y$ plane, the trap being $\sim 5 \%$ stiffer in the (polarization) $y$ direction. The potential in the $x-z$ plane is only moderately elongated, as a consequence of the relatively high axial trap stiffness.

Table 1 summarizes the experimental results obtained on 15 different measurements involving three different micromirrors. It also shows the results of a series of measurements made for comparison on a conventional OT, where the trap was generated through a water-immersion microscope objective (Zeiss C-Apochromat $40 \times / 1.20 \mathrm{~W}$ Corr UV-VIS-IR). Measurements were performed in analog conditions (same PS spheres, trapping laser, and detection system, similar beam to entrance-pupil overfilling ratio,
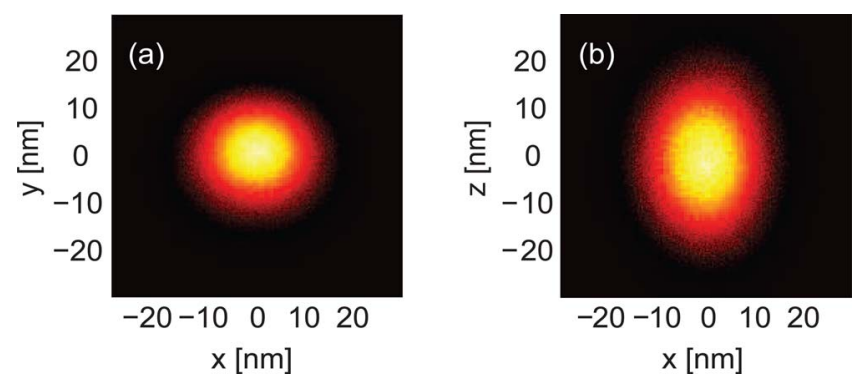

Fig. 3. (Color online) Histograms showing the experimental position distributions of a particle trapped in an MOT during $20.2 \mathrm{~s}$ (a) in the $x-y$ plane and (b) in the $x-z$ plane. 
Table 1. Average Transverse Trap Stiffness $\kappa_{x y}=\left(\kappa_{x}\right.$ $\left.+\kappa_{y}\right) / 2$ and Axial Trap Stiffness $\kappa_{z}$, Normalized by the Effective Optical Power $P_{\text {trap }}$ in the Trap (Units are $\mu \mathrm{N} / \mathrm{m} / \mathrm{mW}$ ), and Ratio $\kappa_{x y} / \kappa_{z}$ Between Transverse and Axial Trap Stiffnesses (Dimensionless) $^{a}$

\begin{tabular}{ccc}
\hline & $\begin{array}{c}\text { Micromirror } \\
(n=15)\end{array}$ & $\begin{array}{c}\text { Objective } \\
(n=6)\end{array}$ \\
\hline$\kappa_{x y} / P_{\text {trap }}$ & $1.20 \pm 0.03$ & $1.11 \pm 0.03$ \\
$\kappa_{z} / P_{\text {trap }}$ & $0.52 \pm 0.02$ & $0.37 \pm 0.01$ \\
$\kappa_{x y} / \kappa_{z}$ & $2.31 \pm 0.10$ & $3.00 \pm 0.06$ \\
\hline
\end{tabular}

${ }^{a}$ Values measured on the MOT are compared to values measured on conventional OT formed through a high-NA microscope objective. Errors indicate the standard deviation on $n$ individual measurements.

identical power accounting for the measured objective transmission of $60 \%$ at the laser wavelength). The measured values of the transverse trap stiffness, normalized by the effective optical power in the trap, are closely matching the theoretical and experimental value of $\sim 1(\mu \mathrm{N} / \mathrm{m}) / \mathrm{mW}$ previously reported with a high-NA microscope objective for $1 \mu \mathrm{m}$ radius PS spheres [17]. The stiffnesses measured on the MOT are even superior to those measured on the conventional OT, especially in the $z$ direction. While a systematic underestimation of optical power in the MOT may have resulted in a small overestimation of the force per unit optical power, the ratio $\kappa_{x y} / \kappa_{z}$ between the transverse and axial trap stiffnesses is independent of the optical power. Thus this ratio clearly demonstrates that micromirrors generate traps with a proportionally higher axial stiffness. At first glance, these results may seem surprising, as the micromirrors used in the present experiments have an NA of 0.93 , lower than the $1.2 \mathrm{NA}$ of the microscope objective. However, the focusing characteristics of a micromirror are different from that of a microscope objective. Indeed, the (intensity) apodization function of a microscope objective obeying the sine condition is given by $A(\theta)=\cos \theta$, which approaches zero for large focusing angles. In contrast, a parabolic micromirror is characterized by $A(\theta)=4 /(1+\cos \theta)^{2}$, which diverges for large angle rays (a derivation can be found in [18]). A micromirror thus naturally generates a focused beam with proportionally more energy in its higher spatial frequency components, which is known to play an important role in the axial trap stiffness $[19,20]$. Apart from differences in the apodization, there might also be one in the amount of spherical aberration (SA). SA generated by the micromirrors depends on the respective thicknesses of the glass and epoxy layers and the trapping depth, as well as on the accurate cross-sectional profile of micromirrors, which is still to be investigated in detail.

In conclusion, we successfully measured the 3D trap stiffness of micromirror optical tweezers using back-focal-plane interferometry. It is shown that micromirrors can generate trap stiffnesses that are even superior to those achieved with a microscope objective, especially in the axial direction. In principle, micromirrors may be also used for simultaneously collecting backscattered light for position tracking, leaving behind the need for a condenser objective. Miniaturized systems enabling fluctuation measurements from multiple micromirrors may be achieved in this manner.

\section{References}

1. A. Ashkin, J. M. Dziedzic, J. E. Bjorkholm, and S. Chu, Opt. Lett. 11, 288 (1986).

2. S. M. Block, L. S. B. Goldstein, and B. J. Schnapp, Nature 348, 348 (1990).

3. T. T. Perkins, S. R. Quake, D. E. Smith, and S. Chu, Science 264, 822 (1994).

4. B. Lukić, S. Jeney, C. Tischer, A. J. Kulik, L. Forró, and E. L. Florin, Phys. Rev. Lett. 95, 160601 (2005).

5. G. M. Wang, E. M. Sevick, E. Mittag, D. J. Searles, and D. J. Evans, Phys. Rev. Lett. 89, 050601 (2002).

6. C. Guzman, H. Flyvbjerg, R. Koszali, C. Ecoffet, L. Forró, and S. Jeney, Appl. Phys. Lett. 93, 184102 (2008).

7. F. Merenda, J. Rohner, J. M. Fournier, and R. P. Salathé, Opt. Express 15, 6075 (2007).

8. A. Constable, J. Kim, J. Mervis, F. Zarinetchi, and M. Prentiss, Opt. Lett. 18, 1867 (1993).

9. Z. H. Liu, C. K. Guo, J. Yang, and L. B. Yuan, Opt. Express 14, 12510 (2006).

10. C. Liberale, P. Minzioni, F. Bragheri, F. De Angelis, E. Di Fabrizio, and I. Cristiani, Nat. Photonics 1, 723 (2007).

11. S. J. Cran-McGreehin, K. Dholakia, and T. F. Krauss, Opt. Express 14, 7723 (2006).

12. E. Schonbrun, C. Rinzler, and K. B. Crozier, Appl. Phys. Lett. 92, 071112 (2008).

13. L. P. Ghislain, N. A. Switz, and W. W. Webb, Rev. Sci. Instrum. 65, 2762 (1994).

14. A. Pralle, M. Prummer, E. L. Florin, E. H. K. Stelzer, and J. K. H. Hörber, Microsc. Res. Tech. 44, 378 (1999).

15. K. Berg-Sørensen and H. Flyvbjerg, Rev. Sci. Instrum. 75, 594 (2004).

16. B. Lukic, S. Jeney, Z. Sviben, A. J. Kulik, E. L. Florin, and L. Forró, Phys. Rev. E 76, 011112 (2007).

17. N. B. Viana, A. Mazolli, P. A. M. Neto, H. M. Nussenzveig, M. S. Rocha, and O. N. Mesquita, Appl. Phys. Lett. 88, 131110 (2006).

18. J. J. Stamnes, Waves in Focal Regions (Hilger, 1986), Sec. 16.1.2.

19. A. Ashkin, Biophys. J. 61, 569 (1992).

20. N. B. Simpson, D. McGloin, K. Dholakia, L. Allen, and M. J. Padgett, J. Mod. Opt. 45, 1943 (1998). 